\title{
Real-time underwater object detection based on an electrically scanned high- resolution sonar
}

\author{
Henriksen, Lars
}

Published in:

Proceedings of the Symposium on Autonomous Underwater Vehicle Technology

Link to article, DOI:

10.1109/AUV.1994.518613

Publication date:

1994

Document Version

Publisher's PDF, also known as Version of record

Link back to DTU Orbit

Citation (APA):

Henriksen, L. (1994). Real-time underwater object detection based on an electrically scanned high-resolution sonar. In Proceedings of the Symposium on Autonomous Underwater Vehicle Technology (pp. 99-104). IEEE. https://doi.org/10.1109/AUV.1994.518613

\section{General rights}

Copyright and moral rights for the publications made accessible in the public portal are retained by the authors and/or other copyright owners and it is a condition of accessing publications that users recognise and abide by the legal requirements associated with these rights.

- Users may download and print one copy of any publication from the public portal for the purpose of private study or research.

- You may not further distribute the material or use it for any profit-making activity or commercial gain

- You may freely distribute the URL identifying the publication in the public portal 


\title{
Real-time Underwater Object Detection Based on an Electrically Scanned High-resolution Sonar
}

\author{
Lars Henriksen \\ Institute of Automatic Control Systems \\ Technical University of Denmark \\ Building 326, DK-2800 Lyngby, Denmark, e-mail: ho@sl.dth.dk
}

\begin{abstract}
The paper describes an approach to real time detection and tracking of underwater objects, using image sequences from an electrically scanned high-resolution sonar. The use of a high resolution sonar provides a good estimate of the location of the objects, but strains the computers on board, because of the high rate of raw data. The amount of data can be cut down by decreasing the scanned area, but this reduces the possibility of planning an optimal path. In the paper methods are described, that maintains the wide area of detection, without significant loss of precision or speed. This is done by using different scanning patterns for each sample. The detection is based on a two level threshold, making processing fast. Once detected the objects are followed through consecutive sonar images, and by use of an observer the estimation errors on position and velocities are reduced. Intensive use of different on-board sensors also makes it possible to scan a map of a larger area of the seabed in world coordinates. The work is in collaboration with partners under MAST-C T90-0059.
\end{abstract}

\section{INTRODUCTION}

When an AUV is operating in an unknown environment, the success of the mission is highly dependent of an obstacle avoidance system (OAS). This system consists of a pathplanner and an object detection system (ODS), which is the part that will be addressed in this paper.

The task of the ODS is to provide information about position, velocity and size of objects in the near surroundings of the AUV. These data should be available sufficiently early for the pathplanner to generate an evasion manoeuvre. This means that the range of the sonar sensor should be large enough to allow initial detection of the object, processing of several consecutive images to reduce uncertainty on measured values, and to allow room for the AUV evasion manoeuvre with a safe margin between the AUV and the object.

At the same time the precision of the required data should be high, in order to generate the best path. These two goals are conflicting, as sonar resolution decreases with larger range.
Many of the applications presentcd in the literature have been based on sonars with very low resolution in both range and in horizontal and vertical directions. This yields very rough estimates of the positions and even worse estimates of the velocities of detected objects. This is inconvenient because the output of sonar and the detection algorithm is a part of the information available to the pathplanner. Given imprecise position and velocity of a set of obstacles the planner have to generate a path with a relatively big margin between the AUV and obstacles. This results in an increased number of tums and sometimes also sharper turns, which all consumes energy, which is limited. Use of a high resolution sonar offers the possibility to reduce these unwanted effects that are due to low precision. The fact that a wide range of commercially available sonars offers high resolution without need for external equipment, makes this kind of sonar attractive for especially AUV applications.

The basic problem in using high resolution sonars is that the output is much more complex than for low resolution sonars. Whereas the output from a low resolution sonar is suitable for a simple avoidance reflex but not a real pathplanning, the high resolution sonar produces an image that can be compared to that of a videocamera in complexity. This demands more advanced algorithms for both interpretation of the output, as well for navigation. At the same time the rate of which the data are available, is very high. An output data rate of approx. $1 \mathrm{Mb} / \mathrm{sec}$. is not unusual. These two facts, a lot of data and a complex processing of these, indicate that a selection of relevant data has to be done before processing of data, in order not to load the on board computer to a degree, where real time processing no longer is possible.

\section{A. Target applicalion.}

The target application of the object detection system is an AUV called MARIUS operating close to the seabed at depths at approx. $600 \mathrm{~m} \mathrm{[5]} \mathrm{and} \mathrm{[6].} \mathrm{The} \mathrm{cruising} \mathrm{speed} \mathrm{is} \mathrm{approx.} 2$ $\mathrm{m} / \mathrm{s}$ for one hour. The movements of the AUV are measured by 
various sensors on board (e.g. Gyros, accelerometers and audible contact with fixed transponders), yielding position and orientation in world coordinates through a kalman filter.

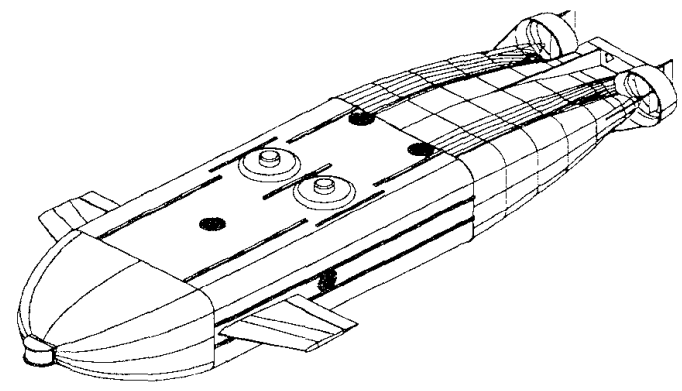

Figure 1. Target vehicle MARIUS.

\section{B. Target sonar}

The sonar used is a SEABAT 6012 high resolution sonar operating at $455 \mathrm{KHz}$ [7]. The image is divided horizontally in 60 beams, each with a width of $1.5^{\circ}$, covering $90^{\circ}$ in front of the sonar. Each beam is sampled to a resolution of $5 \mathrm{~cm}$. Vertically the image has only one plane covering $\pm 10^{\circ}$. The range of the sonar is adjustable up to $200 \mathrm{~m}$. The sampling rate is 0.9 $\mathrm{MB} / \mathrm{sec}$, which yields an image update frequency of approx. 7 images per second at a range of $100 \mathrm{~m}$. Later versions of the sonar will have 3 beams in the vertical direction. The results in this paper can easily be extended to this case.

\section{Selection of Raw sonar data}

The amount of data processing available for each echo sample is limited. Because of the high rate of raw sonar data actions must be taken to minimize the complexity of the employed algorithms, and/or reduce the effective rate of the image data, without losing relevant information. The latter will be discussed in this section and the first in the next section.

\section{A. Goals and apriori knowledge.}

When reducing data there are basically two goals to achieve: First new objects should be detectable at an early stage, i.e. at long range to ensure time for processing and manoeuvring. Second already detected objects should be tracked for as many samples as possible, in order to obtain precise estimates. This ensures sufficient information for the pathplanner. The apriori knowledge about the objects that can be encountered is limited. They may appear from every direction at every point in the sonar image, which indicates that all of the image should be processed. The velocity can be quite high if objects are floating with the current straining the tracking algorithm.

\section{B. Ignore background}

When operating close to the sea bottom the surface may cause echoes of significant strength. These are spread out over a larger portion of the sonar image, hiding echoes from real objects. Processing such an area demands quite advanced signal processing, which consumes much time. It is therefore attractive to discard processing of such an area, and wait until the object reaches a part of the image less noise corrupted. The areas with botton reverberation are easily detected by a high variance.

\section{Subsampling in time and position}

A fast and simple method of reducing data is to subsample the sonar image. This can be done either temporally or spatially. If the subsampling is done in the time domain only every $n$ 'th image is processed, leaving more time for each image on the computer. The negative consequences are a deteriorated precision of the estimates due to the fewer updates to the observer, and a longer response time to new obstacles. Care should be taken not to subsample so much that time necessary for processing and evading is exceeded. Expression (1) shows the allowed subsampling $n_{\max }$ as a function of the sonar image frequency $f_{\text {sonar }}$ the number of images input to the observer $N_{\text {pic }}$, the AUV speed $V$, detection distance $D_{\text {det }}$, and the required distance for manocuvring $\mathrm{D}_{\text {manoeuver }}$

$$
n_{\text {max }}=\frac{f_{\text {sonar }}}{V N_{\text {pic }}}\left(D_{\text {det }}-D_{\text {manoetuver }}\right)
$$

If $n$ is kept below $n_{\max }$ the system is running real time since the bandwidth of processing is bigger than that of the system controlled.

When subsampling spatially it is attractive to process every n'th range in the image (range quantization). This reduces the data to $1 / \mathrm{n}$ 'th, but also increases the minimum size of detectable objects to $\mathrm{nR}$, where $\mathrm{R}$ is the standard range resolution. Furthermore small echoes may vanish in the 'holes' between the scanned ranges. This may be fatal for the mission.

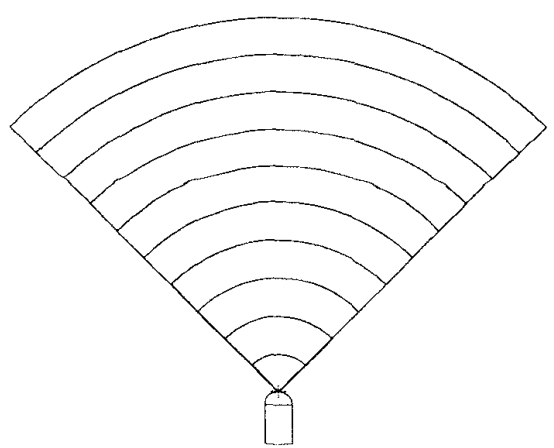

Figure 2. Subsampling in range. 
It is not attractive to subsample the norizontal beams as these cover a very wide area at long range, even with a very high horizontal resolution $(2.6 \mathrm{~m}$ with target sonar at $100 \mathrm{~m}$ range compared with $5 \mathrm{~cm}$ range resolution). The subsampling should be implemented in a way that ensures inspection of all pixels every $n$ images. This avoids that no object will be undetected, if the AUV and the objects all are at fixed locations.

\section{Area of interest, multirangeimultirate approach}

The purpose of the object detection is to provide input to a pathplanner enabling it to avoid upcoming obstacles. This information should be available sufficiently early, to ensure a proper safe distance between AUV and object. As time to contact decreases proportional with distance to object it is indicated, that the parts of the sonar image close to the AUV is of more importance than parts further away for the avoidance procedure. This area should therefore be processed frequently. However scanning only short range in front of the AUV will decrease the possibility to plan the best long term path forward. Processing a larger part of the image once in a while will ensure the necessary long range information.

A way to meet these partial goals is the multirange/multirate approach. In this the sonar image is divided into a number of partially overlapping regions, which all cover the full horizontal view. The regions start at the range zero and ends at multiple ranges between zero and maximum range as seen on figure 3.

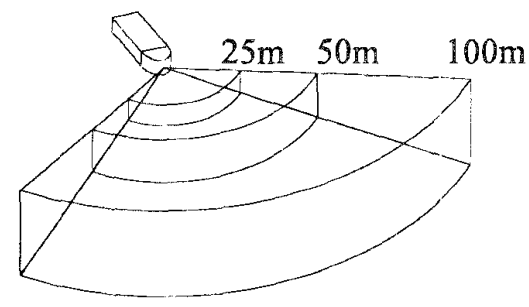

Figure 3. Multirange/multirate processing.

The processing frequency of each area is a function of the size of the area in bytes, and the importance to the avoidance algorithm. This function is to be designed so that it is ensured, that the image processing computer is evenly loaded over time. The principle is illustrated by an example. We have divided the image into three areas $\mathrm{A}, \mathrm{B}$ and $\mathrm{C}$ defined by three ranges 25 , 50 and $100 \mathrm{~m}$. With the relative importance of the areas appraised to $4: 2: 1$ the situation is illustrated on figure 4 . Here one increment in time corresponds to the time consumed by processing $25 \mathrm{~m}$ of range in the image.

The processing pattern is repeated every 8 time instances. It is seen that with the same overall load of the computer the range $0-25 \mathrm{~m}$ is processed 4 times per cycle, instead of 2.5

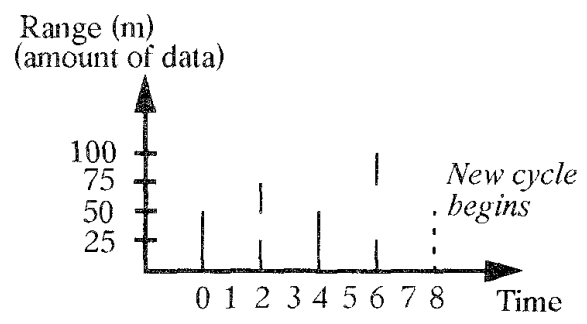

Figure 4. Multirate processing.

times that would be the case if the entire range was processed every time. This improvement is at the expense of e.g. Only one scan of the range $50-100 \mathrm{~m}$ per cycle, against 2.5 times at standard processing pattern. The multirange/multirate approach has in this way assigned processing power to the different areas of the image, in accordance to the interest for the application.

\section{E. Multirange/multidirection}

Another method is the multirange/multidirection approach. This utilizes that the AUV for object avoidance purposes only need information about objects near the path of the vehicle. This information about the future movements of the AUV is readily available from the pathplanner.

Dilating the path with the safety radius of the AUV defines the area to be processed. This area of interest is not well suited for representation in polar coordinates, and this imposes some difficulties for the practical implementation, as the sonar image is polar by disposition. These difficulties can be overcome by approximating (discretizising) the area of interest to a number of range-, width areas of the sonar. Processing these sequentially ensures full coverage of the dilated path, when discretization is made with enough margin. The principle is illustrated on figure 5 , where the safety radius is $20 \mathrm{~m}$. The discretization ranges and widths are $0-25 \mathrm{~m} \mathrm{90}, 25-50 \mathrm{~m} \mathrm{45^{ \circ }}$. and $50-100 \mathrm{~m}$ $22.5^{\circ}$.

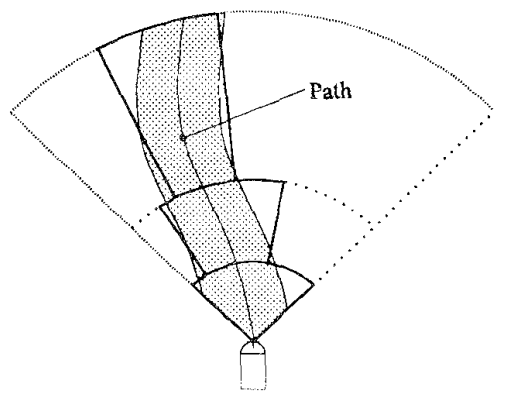

Figure 5. Multirange/multdirection processing.

The processing corresponding to this area is $50 \%$ of the entire image. The rate at which data are ready can in this way 
be doubled, compared to full range processing, without losing important information.

\section{F. Combination of principles}

The principles described can be merged with favourable results. The main algorithm is the multirange/multidirection method, as this covers the area of principal interest for object avoidance purposes, and provides the fastest information update frequency. This algorithm can be enhanced by adding the multirate technique allocating more time to the area right in front of the AUV. This is supplemented by a series of full width multirange scan sequences, at a low frequency. Output from this processing does not contribute to avoidance performance, but provides information necessary for the path planner in order to generate an optimal path, if an object should demand deviation from the originally planned path. The advantages of the methods are briefly summarized in table 1.

TABLE 1. Data selection approaches

\begin{tabular}{l|l|l|} 
& Principle & Features \\
\hline $\begin{array}{l}\text { Multi- } \\
\text { range,- } \\
\text { rate }\end{array}$ & $\begin{array}{l}\text { Computer power } \\
\text { allocated to most } \\
\text { important areas of } \\
\text { sonar image. }\end{array}$ & $\begin{array}{l}\text { Near range fre- } \\
\text { quently processed. } \\
\text { Full range scan for } \\
\text { pathplanner. }\end{array}$ \\
\hline $\begin{array}{l}\text { Multi- } \\
\text { range, } \\
\text { direction }\end{array}$ & $\begin{array}{l}\text { Only relevant path } \\
\text { processed. }\end{array}$ & $\begin{array}{l}\text { Fast update of path. } \\
\text { No update of other } \\
\text { areas of image }\end{array}$ \\
\hline $\begin{array}{l}\text { Com- } \\
\text { bined } \\
\text { method }\end{array}$ & $\begin{array}{l}\text { Main scan: Only rel- } \\
\text { evant path pro- } \\
\text { cessed, Near range } \\
\text { most frequently pro- } \\
\text { cessed. } \\
\text { Batch scan: full } \\
\text { range scan. }\end{array}$ & $\begin{array}{l}\text { Fast update of path, } \\
\text { especially near range. } \\
\text { Scan of full range } \\
\text { and width at low fre- } \\
\text { quency for pathplan- } \\
\text { ner. }\end{array}$ \\
\hline
\end{tabular}

The combined method used in the project has a full range scan frequency which depends on the presence of obstacles,

Besides the processing patterns described above each detected obstacie initiates a small area around it, which is processed at every time instant, disregarding other scanning rules. As the area is small and the number of objects presumably is low, the load on the image processing computer from this task is nearly negligible.

\section{Obiect DETECTION Aigorithm}

A sonar image differs quite remarkably from an optically recorded picture. First of all it contains information about depth, and can in this way detect a scene $3 \mathrm{D}$ instead of $2 \mathrm{D}$. Secondly it is the only long range sensor feasible for underwater application. Thirdly the sensing process is very complex, resulting in an image quality that is far worse than for optical images. In this section it is described how the negative consequences can be minimized.

\section{A. Problem}

The sensor is active, and the media conveying the information are onnidirectional pressure waves in a liquid. The signal is reflection of waves when it encounters an object. The amplitude of this echo is dependent on the shift in impedance from water to object, and angle of incidence for specular reflection, and surface roughness for diffuse reflection [1]. The echoes are in this way highly dependent on the material, surface and orientation of the object. As this is inherent to the sensing method, no countermeasures can be taken. This is very unfortunate, as an object may return only very weak echoes at a critical point.

Small objects floating in the water, layers of warm and cold water, noise due to the time-varying-gain of the sonar, the signal processing algorithms amongst others produce noise in the picture. This, in combination with low echoes returned from the object, complicates detection.

\section{B. Polar or carthesian processing}

Before choosing a processing algorithm for the detection it should be considered whether the algorithm is to work on the native polar set of data or on carthesian data. The latter has the advantage, that an object described in equisized pixels are relatively independent on where in the image the object is located. Running e.g. a convolution kemel in polar coordinates on the same object, but at different ranges, will result in different output, depending on the range, as the kemel covers from a few $\mathrm{cm}$ to several meters.

On the other hand carthesian processing requires a transformation from polar coordinates which can be a very time consuming task compared with the benefits which are limited. As input is $120 \mathrm{~KB}$ the output is $3.1 \mathrm{MB}$ for a resolution of $5 * 5$ $\mathrm{cm}$. This is 26 times more data. Processing would therefore require a large amount of trigonometric calculations, a look up table of $6.2 \mathrm{MB}$ or some dedicated hardware. Only the latter is feasible for real-time applications. The conversion does not alter the fact, that the information density at long range is very poor, but only allows some ordinary image processing techniques. For most applications these do not improve performance significantly, and it is therefore not attractive to work in carthesian coordinates.

\section{Detection and grouping}

The image from the sonar is highly noise corrupted. This demands either some kind of noise reduction or an algorithm which is robust to the noise encountered. Noise reduction generally takes time. A one dimensional noise reducing kemel as $1,2,1$ needs $3 * 120.000$ additions pr full image before extraction of features. Even with a reduced area of interest subsampling in time may be necessary.

Currently we are using a two level threshold, which has shown to have a good performance. It is a one pixel operator 
with no multiplications, making it quite fast. It does not need any prefiltering improving speed even more. The method is based on the assumption that an object occurring in the image will have an echo significantly above the background noise. We are using a detection level of approximately $60 \%$ of maximum output for classifying a pixel as belonging to an object. Not all the object echos will have strength to exceed the high level. These parts of the object are recognized as they exceed a lower level of threshold, and being adjacant to a pixel classified as an object. In this way detection of an object will propagate from one single pixel with high echo. Setting the lower level to approx. $25 \%$ of maximum shows good results. In this method only the presence of obstacles will cause more than one operation on each pixel, and even then it will only happen in a local area. The result of processing a single beam from a real sonar image recorded at Holmen, DK, is seen on figure 6. Maximum output is 127.

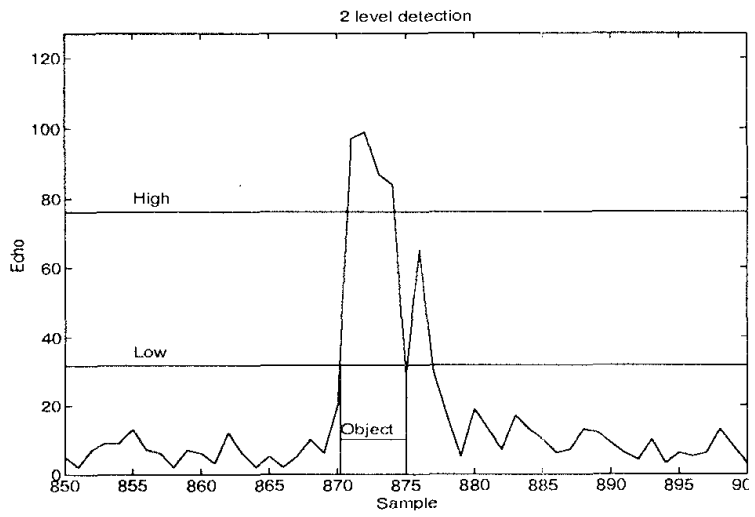

Figure 6. Thresholding in real sonar image (One beam of 60 , Holmen, DK).

On the figure it is seen that the threshold has detected an object at the range $43.6 \mathrm{~m}$, which is the actual location of the test object.

Once the object is located, the size is determined. This for the use of the pathplanner, which has to know the free and occupied space in the surroundings. The size is represented as a circle circumscribing the object in cathesian coordinates, as this is enough for avoiding purposes.

In future development the thresholding is to be extended to adaptive threshold levels. To obtain this median filters are used to remove spikes and object echoes, in order to determine the level the average echo.

\section{Observer/Tracker}

The proper behaviour of the AUV depends on the pathplanner having access to accurate information about the objects in the environment. If this is not the case the shortest path may not be found or collisions may occur.

The output of the object detection algorithm however suffers from noise at some degree. This is due both to the noise in the sonar image, the use of a simplified detection algorithm and the significant discretization of position at long range. A way to reduce this uncertainty is to use an observer. This calculates an optimal estimate of position velocity and size of the objects derected by use of series of consecutive images.

\section{A. Structure}

Available input for the observer is the object coordinates in a bodyfixed coordinate system $z_{o b}$, obtained from the sonar. The position and orientation of the AUV in a global fixed system $z_{a g}$ is known as well. This is the output of a primary Kalman filter based on various sensors, including a set of transponders. This observer is not to be discussed here. The position of the objects in global coordinates $z_{o g}$ can be found by equation (2) where $R$ is the rotation matrix describing the orientation of the AUV.

$$
z_{o g}=z_{a g}+R^{-1} z_{o g}
$$

Representing the objects in world coordinates has the advantage that the observer is linear and thus more simple, and at the same time yielding better estimates. Another benefit is the possibility to scan a large area of the seabed, generating a map of objects for use on future missions.

The state vector for each object consists of position and orientation of the object as described in [2]. This is supplemented by an additional parameter $S_{k r}$ which is the radius of the circle circumscribing the object

$$
S_{k x}=\left[\begin{array}{c}
x_{k} \\
\dot{x}_{k}
\end{array}\right] \quad S_{k y}=\left[\begin{array}{c}
y_{k} \\
\dot{y}_{k}
\end{array}\right] \quad S_{k z}=\left[\begin{array}{c}
z_{k} \\
\dot{z_{k}}
\end{array}\right] \quad S_{k r}=r
$$

Each of these 4 parameters have their own independent Kalman filter. As objects may dis- and reappear in the image eventdriven filters are feasible.

When an object is located the following 5 steps are executed: Time update of state:

$$
S_{k}^{\prime}=\Phi_{k, k-1} \dot{S}_{k-1}
$$

Time update of error covariance matrix

$$
P_{k}^{\prime}=\Phi_{k, k-1} \hat{P}_{k-1} \Phi_{k, k-1}^{T}+Q_{k, k-1}
$$

Calculation of the Kalman gain

$$
K_{k}=P_{k}^{\prime} H^{T}\left(H P_{k}^{\prime} H^{T}+R_{k}\right)^{-1}
$$


The measurement update of state (optimal estimate)

$$
\hat{S}_{k}=S_{k}{ }_{k}+K_{k}\left(\varepsilon_{k}-H S_{k}^{\prime}\right)
$$

and the measurement update of the error covariance matrix (optimal estimate)

$$
\hat{P}_{k}=\left(I-K_{k} H\right) P_{k}^{\prime}
$$

Notation used: $\Phi_{\chi, x-1}$ is the transition matrix, $Q_{x, x-1}$ is covariance matrix of the process noise and $R_{k}$ is covariance of the measurement noise process. ' denotes time update, and ' the optimal estimate. $\Phi_{x, x-1}$ and $H$ for position and velocity are

$$
\Phi_{k, k-1}=\left[\begin{array}{cc}
1 & \Delta T \\
0 & 1
\end{array}\right] \quad H=\left[\begin{array}{ll}
1 & 0
\end{array}\right]
$$

Determining $Q_{k, k-1}$ is the main problem and is subject to investigation.

\section{B. Further tracking}

A detected object will eventually move out of the field of the sonar, due to the movements of AUV and object. At this point the update is switched from Kalman filter to time update only. In this way objects can be tracked even when out of contact. This is of importance if the task of the AUV makes it likely, that it will retum to the same site later.

The estimation precision of the state of the object naturally decreases with time, both due to the initial estimation uncertainty and due to changes in direction and speed of the object. After a number of updates the information is of so little value to the pathplanner, that update is no longer beneficial. At this point the state of the object is frozen and stored together with at time stamp. This ensures that the tracking can be restarted at any time, should it be necessary. Another reason for the hibernation of the state is that unrestricted tracking will concern a constantly increasing number of objects, each consuming processing power. When to hibemate an object mainly depends on the uncertainty of the state of the object. This can be measured by the covariance matrix exceeding some predefined level, or just that a number of updates have passed.

\section{Determination of motion}

For ordinary object avoidance purposes the Kalman filter is as described above. If the task is to scan an area of the seabed it is necessary to determine whether the object is moving or not, as only fixed objects should be mapped. This can be determined by regarding the observed speed, optionally as a worst case by including the variances.

\section{Conclusion}

We have described a way to reduce the need for computer power, by processing areas of the sonar image at a frequency according to importance for the mission. This frees power for more advanced detection algorithms, and for real time processing.

Detection of objects is currently simple yet reliable, with a slight tendency to detect false targets. Only very few objects are missed.

Future work will concentrate on more sophisticated detection algorithms, as of investigation of the possibility to reduce noise by defining some correlation between noise in consecutive images.

\section{ACKNOWLEDGMENTS}

This research was gratefully supported by a grant form the Danish Technical Research Council, STVF (Statens TekniskVidenskabelige Forskningsråd) under license No. STVF 165245-1 OS.

I would like to thank Allan T. Sørensen, who performed much of the programming of our simulator, and made actual recordings with the sonar.

\section{REFERENCES}

[1] D. Langer, M. Herbert. Building elevation maps from underwater sonar data. Proceedings for unmanned untethered submersible technology, 1991, pages 328-341

[2] Glen N. Williams, Glenn E. Lagace, Alfred J. Woodfin. A collision avoidance controller for autonomous underwater vehicles. Proceedings of $A U V$ '90. Pages 206-213.

[3] B. Shridhar, P. Smith, R. Suorsa, B. Hussein. Multirate and event-driven kalman filters for helicopter flight. IEEE Control Systems, vol. 13 No 4 1993. Pages 26-33.

[4] Mark T. Shaw, Obstacle avoidance sonar, An analysis of system requirements and detection performance during near-bottom operation. AUV ' 90 . Pages 223-228

[5] A. Pascoal, A. Bjerrum, J. Coudeville. Marius (Marine Utility System) - An autonomous underwater vehicle for environmental surveying. MArine Science and Technology, MAST Days 1993.

[6] A. Bjerrum, A. Pascoal, J. M. Coudeville, K. Christiansen. Control of an Autonomous Underwater Vehicle for Environmental Surveying (MARIUS). Control Applications in Marine Systems, CAMS 1992.

[7] C. J. Simonsen. Mine locating and disposal, the need for real-time scanning sonars. Presentation made at UDT '93 in Cannes. 